\title{
Optimization of conditions for preparation of poly (vinyl alcohol)-water glass adhesive by response surface methodology
}

\author{
Xinli Zhang ${ }^{\mathrm{a}}$, Qi Chen ${ }^{\mathrm{b}}$ and Ji Wang ${ }^{\mathrm{c}}$ \\ College of Materials Science and Engineering, Central South University of Forestry and \\ Technology, Changsha, 410004, China \\ axinli81@163.com, bcq19931105@icloud.com, cwd_baby@126.com
}

\begin{abstract}
Keywords: wood adhesive; water glass; poly (vinyl alcohol); bonding strength; response surface methodology
\end{abstract}

Abstract. Water glass (WG) has potential applications in many fields, but the poor water-resistance limits its further development. In the article, a kind of modified WG adhesive was designed from WG and poly (vinyl alcohol) (PVA), in order to improve the bonding properties between WG adhesive and poplar veneer. Preparation conditions of PVA-WG adhesive were optimized by response surface method. Based on the single factor experiments, the optimum values of the parameters were obtained by Box-Behnken design using the Design-Expert software. The results showed that the important parameters influencing the bonding strength were the mass ratio of PVA to WG, dosage of sodium tartaric acid (TA) and the aging time. The optimum technical conditions were the mass ration of PVA to WG 0.65 , dosage of TA $1.00 \mathrm{~g}$ and the aging time $5.00 \mathrm{~h}$. At this condition, bonding strength of as-prepared PVA-WG adhesive could reach $1.0407 \mathrm{MPa}$, close to the actual value of $1.01 \mathrm{MPa}$, so the model fits pretty well. The as-prepared PVA-WG adhesive had better adhesion properties to poplar veneer, which is meaningful for developing environmental friendly adhesive to replace organic adhesive in the wood-based panel field.

\section{Introduction}

As is known to all, the industry of wood-based panel has a huge demand for adhesive. The properties of adhesive directly determine the quality and application of the wood-based panel and affect the living environment of people. For a long time, organic adhesive (mainly urea-formaldehyde, phenolic, and melamine formaldehyde resin) are used in the field of wood-based panel although they have the problem of environmental pollution ${ }^{1]}$. In recent years, some environmental friendly wood adhesive, such as $\operatorname{starch}^{[2]}$, soybean protein ${ }^{[3]}$, mussel protein ${ }^{[4]}, \operatorname{tannin}^{[5]}$, $\operatorname{konjac}^{[6]}$, $\operatorname{lignin}^{[7]}$, and waterborne isocyanate ${ }^{[8]}$, etc., became popular and developed for the sake of human health. But the low bonding strength, poor water resistance and heat resistance are widespread for the adhesive prepared from natural renewable resources ${ }^{[9]}$. Some waterborne polymer adhesive has good bonding performance to wood, but the cost of preparation is high, which is not conducive to large-scale production.

Water glass (WG, water solution of sodium silicate) has long been used as dependable, low cost adhesive for bonding a variety of porous surfaces and materials such as paper, soil, mineral wool, mica and wood ${ }^{[10]}$. WG is odorless, nonflammable, and recognized as safe, but cured WG is brittle and inelasticity, moreover, its water resistance is very poor. There are many ways for WG modification to improve its properties ${ }^{[11]}$. However, the modification of WG with PVA by forming a cross-linked network and the optimized preparation technology of it has not been reported.

Poly (vinyl alcohol) (PVA) was selected to modified WG in order to form a appropriate interpenetrating network between them by cross-linking with strong acid and weak acid step by step in this study. On the basis of the previous single factor experiment, the preparation technology of the PVA-WG adhesive was optimized using Box-Behnken experiment design module by response surface methodology, with the aim to provide theoretical basis and practical reference for obtaining WG wood adhesive with excellent bonding performance. 


\section{Experimental}

Materials. PVA (17-99) was supplied by Yunnan Yunwei Co., Ltd. (China). WG (3.2 modulus, $42^{\circ}$ Bé, $\mathrm{pH}=10.8$ ) was purchased from Shangyu Qiangsheng Chemical Co., Ltd (China). SDS and TA were purchased from Tianjin Fuchen Chemical Reagent Factory (China), and boric acid (BA) was from Tianjin Hengxing Chemical Reagent Factory. All the reagents were analytical grade. Fast-growing poplar was obtained from Yiyang city in Hunan province (China), the moisture content of poplar veneer was $12.1 \% \pm 0.1 \%$, and its thickness was $0.2 \mathrm{~mm}$.

Preparation of PVA-WG adhesive. PVA-WG adhesive was prepared as follows: two-thirds of the total of WG, one-thirds of total distilled water and SDS were added in a $500 \mathrm{ml}$ three-necked round bottom flask equipped with a electric mixer and a thermometer, then continuously stirred for 30 min to form a emulsion. A certain of PVA solution $(10 \mathrm{wt}, \%)$ was added and mixed. When the temperature of the flask was $80^{\circ} \mathrm{C}$, one-thirds of total distilled water and TA was dropped into the flask over a period of $1 \mathrm{~h}$. After that, the rest of WG and distilled water was added into the flask over a period of $1 \mathrm{~h}$. Finally, BA was added and stirred for $20 \mathrm{~min}$. After a period of time of insulation reaction of the mixture at $40{ }^{\circ} \mathrm{C}$, the product of PVA-WG adhesive was obtained.

Preparation of glued specimen and bonding strength test. The PVA-WG adhesive was evenly coated on the surface of poplar veneer (with the dimension of $300 \mathrm{~mm} \times 300 \mathrm{~mm}$ ) by the way of manual brushing, using the conventional way of vertical texture group into three-layers of plywood with the dosage of the adhesive of $250-280 \mathrm{~g} \cdot \mathrm{m}^{-2}$. The wet plywood was placed on a press machine at $70{ }^{\circ} \mathrm{C}$ for $20 \mathrm{~min}$ with the pressure of $0.80 \mathrm{MPa}$. The three-layers plywood was placed at room temperature for 7 days, then was sawed into the test specimen according to GB/T 17659-1999 (China).

The bonding strength of poplar plywood was determined using an MWD-50 microcomputer controlled universal mechanical testing machine (Jinan wood-based panel factory, Shandong province, China) according to GB/T 14074.10-2006. The testing speed is $2 \mathrm{~mm} \cdot \mathrm{min}^{-1}$. All the tests were replicated three times, and the results were presented as the averages.

Characterization of PVA-WG adhesive. FTIR spectra were obtained using an IRAffinity-1 spectrometer (Shimadzu, Japan) with samples in $\mathrm{KBr}$ pellets. Each sample was scanned 32 times over a region of $4000-400 \mathrm{~cm}^{-1}$ at a resolution of $4 \mathrm{~cm}^{-1}$. Specimens were observed using a SEM (FEI Quanta 450) under high vacuum mode. Thermal properties were analyzed using an STA449 F3 Jupiter synchronous thermal analyzer (NETZSCH, Germany). Samples were heated from $40{ }^{\circ} \mathrm{C}$ to $800{ }^{\circ} \mathrm{C}$ at a rate of $20^{\circ} \mathrm{C} \cdot \mathrm{min}^{-1}$ under nitrogen gas flowing at $20 \mathrm{ml} \cdot \mathrm{min}^{-1}$.

Design of experiments. The experimental design was done using the Design-Expert software in design mode of Box-Behnken. The three important factors, mass ratio of PVA to WG $\left(\mathrm{X}_{1}\right)$, dosage of TA $\left(\mathrm{X}_{2}\right)$ and aging time $\left(\mathrm{X}_{3}\right)$, affecting the bonding strength were as the independent variable, the range of three factors was 0.45-0.85, 0.80-1.20 g and 3-5 h, respectively, as shown in Table 1.

Table 1 Factors and levels of Box-Behnken design

\begin{tabular}{ccccc}
\hline Symbol & Independent variable factor & \multicolumn{3}{c}{ Coding and level } \\
\cline { 3 - 5 } & & -1 & 0 & 1 \\
\hline $\mathrm{X}_{1}$ & Mass ratio of PVA to WG & 0.45 & 0.65 & 0.85 \\
$\mathrm{X}_{2}$ & Dosage of TA $(\mathrm{g})$ & 0.80 & 1.00 & 1.20 \\
$\mathrm{X}_{3}$ & Aging time $(\mathrm{h})$ & 3.00 & 5.00 & 7.00 \\
\hline
\end{tabular}

\section{Results and discussion}

Response surface experimental design and results. There are 17 experiments for three factors and three levels of Box-Behnken design, as shown in Table 2. Among them, five experiments coded as 0 are the center of experiments used to estimate the error by the system. 
Table 2 The experimental program and test results

\begin{tabular}{|c|c|c|c|c|c|}
\hline \multirow[b]{2}{*}{$\begin{array}{l}\text { Test } \\
\text { No. }\end{array}$} & \multicolumn{3}{|c|}{ Symbol } & \multicolumn{2}{|c|}{ Response Value Y } \\
\hline & $\mathrm{X}_{1}$ & $\mathrm{X}_{2}$ & $X_{3}$ & $\begin{array}{l}\text { Bonding strength } \\
(\mathrm{MPa})\end{array}$ & $\begin{array}{l}\text { Predictive value of } \\
\text { regression equation } \\
(\mathrm{MPa})\end{array}$ \\
\hline 1 & -1 & -1 & 0 & 0.66 & 0.67 \\
\hline 2 & 1 & -1 & 0 & 0.58 & 0.57 \\
\hline 3 & -1 & 1 & 0 & 0.53 & 0.54 \\
\hline 4 & 1 & 1 & 0 & 0.81 & 0.80 \\
\hline 5 & -1 & 0 & -1 & 0.44 & 0.43 \\
\hline 6 & 1 & 0 & -1 & 0.72 & 0.72 \\
\hline 7 & -1 & 0 & 1 & 0.63 & 0.63 \\
\hline 8 & 1 & 0 & 1 & 0.50 & 0.51 \\
\hline 9 & 0 & -1 & -1 & 0.88 & 0.88 \\
\hline 10 & 0 & 1 & -1 & 0.84 & 0.84 \\
\hline 11 & 0 & -1 & 1 & 0.79 & 0.79 \\
\hline 12 & 0 & 1 & 1 & 0.93 & 0.93 \\
\hline 13 & 0 & 0 & 0 & 1.14 & 1.03 \\
\hline 14 & 0 & 0 & 0 & 0.95 & 1.03 \\
\hline 15 & 0 & 0 & 0 & 1.03 & 1.03 \\
\hline 16 & 0 & 0 & 0 & 1.06 & 1.03 \\
\hline 17 & 0 & 0 & 0 & 0.99 & 1.03 \\
\hline
\end{tabular}

Established experimental model. Analysis of variance (ANOVA) of the four kinds of regression model for the relationship of between three factors are listed in Table 3. Generally, it is significant when p-value $<0.05$ in statistical analysis. Quadratic model fits significantly as can be seen from Table 3, and the adjusted $\mathrm{R}^{2}$ value significantly is higher than other models, so it is recommended using quadratic polynomial model.

Table 3 Comprehensive analysis of the four kinds of regression model

\begin{tabular}{ccccccc}
\hline Type & p-value & $\begin{array}{c}\text { Lack of fit } \\
\mathrm{p} \text {-value }\end{array}$ & $\mathrm{R}^{2}$ & Adjusted $\mathrm{R}^{2}$ & Predicted $\mathrm{R}^{2}$ & Evaluation \\
\hline Linear & 0.9421 & 0.0105 & 0.0286 & -0.1956 & -0.6132 & \\
2FI & 0.7231 & 0.0067 & 0.1440 & -0.3695 & -1.6508 & \\
Quadratic & $<0.0001$ & 0.9931 & 0.9701 & 0.9317 & 0.9448 & Suggested \\
Cubic & 0.9931 & & 0.9707 & 0.8829 & & Aliased \\
\hline
\end{tabular}

Building and testing of the regression equation. According to the experimental results in Table 2 , the fitting equation can be obtained from regression analysis of the data with the bonding strength as the response value.

$Y=1.03+0.044 X_{1}+0.025 X_{2}-0.00375 X_{3}+0.090 X_{1} X_{2}-0.10 X_{1} X_{3}$

$+0.045 X_{2} X_{3}-0.34 X_{1}^{2}-0.051 X_{2}^{2}-0.12 X_{3}^{2}$

The

coefficient of determination $\mathrm{R}^{2}$ is $97.01 \%$, a good description of the fitting equation, which means less than $3 \%$ of the value of the bonding strength cannot be explained by the model.

ANOVA result is listed in Table 4, values of 'p-value' $<0.05$ indicates model terms are significant, the $\mathrm{p}$-value of the model is 0.0002 , far less than 0.05 , so the quadratic polynomial model is significant. In this case, $\mathrm{X}_{1} \mathrm{X}_{2}, \mathrm{X}_{1} \mathrm{X}_{3}, \mathrm{X}_{1}{ }^{2}, \mathrm{X}_{3}{ }^{2}$ are significant model terms, it is not a simple linear relationship between experimental values and response factor, so the best preparation conditions can be obtained by the regression equation. 
Table 4 ANOVA for regression equation

\begin{tabular}{cccccc}
\hline Source & Sum of squares & Degree of freedom & Mean squares & F value & p-value \\
\hline Model & 0.69 & 9 & 0.077 & 25.27 & 0.0002 \\
$\mathrm{X}_{1}$ & 0.015 & 1 & 0.015 & 5.02 & 0.0600 \\
$\mathrm{X}_{2}$ & 0.005 & 1 & 0.005 & 1.64 & 0.2412 \\
$\mathrm{X}_{3}$ & 0.0001125 & 1 & 0.0001125 & 0.037 & 0.8531 \\
$\mathrm{X}_{1} \mathrm{X}_{2}$ & 0.032 & 1 & 0.032 & 10.63 & 0.0139 \\
$\mathrm{X}_{1} \mathrm{X}_{3}$ & 0.042 & 1 & 0.042 & 13.78 & 0.0075 \\
$\mathrm{X}_{2} \mathrm{X}_{3}$ & 0.0081 & 1 & 0.0081 & 2.66 & 0.1472 \\
$\mathrm{X}_{1}{ }^{2}$ & 0.48 & 1 & 0.48 & 157.98 & $<0.0001$ \\
$\mathrm{X}_{2}{ }^{2}$ & 0.011 & 1 & 0.011 & 2.56 & 0.1013 \\
$\mathrm{X}_{3}{ }^{2}$ & 0.064 & 1 & 0.064 & 20.98 & 0.0025 \\
Residual & 0.021 & 7 & 0.003049 & & \\
Lack of fit & 0.000425 & 3 & 0.0001417 & 0.027 & 0.9931 \\
Pure error & 0.021 & 4 & 0.00523 & & \\
Total & 0.71 & 16 & & & \\
\hline
\end{tabular}

Interaction analysis. The three-dimensional response surface and contour plots can convey the three main areas of information: firstly it can directly reflect the degree of influence of each variable on the response value; the second is to reveal the interaction between the factors. Finally, the contour plot reflects the significant degree of interaction between two variables ${ }^{[12]}$. Circular contour indicates weak interactions, oval contour shows strong interactions.

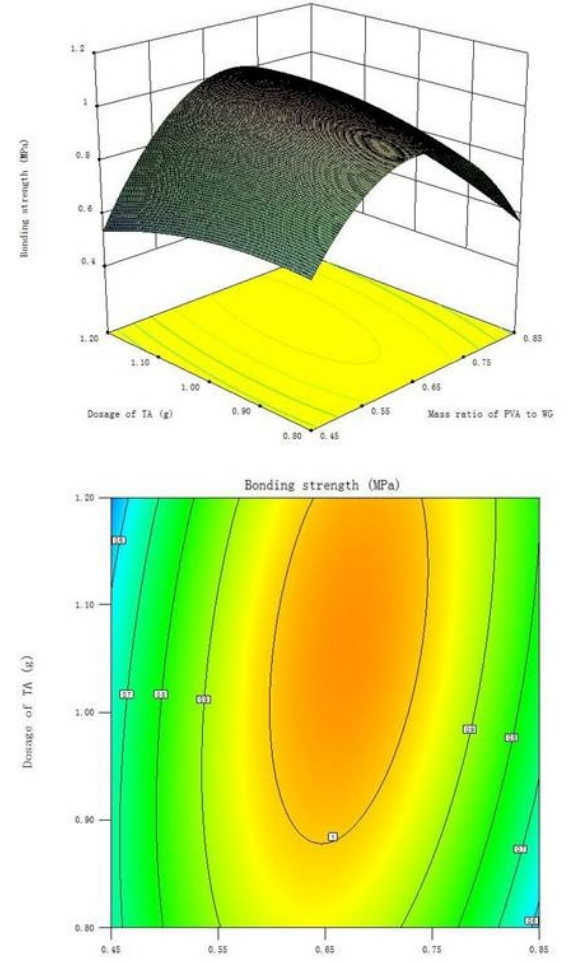

(a) $\mathrm{X}_{1}$ and $\mathrm{X}_{2}$
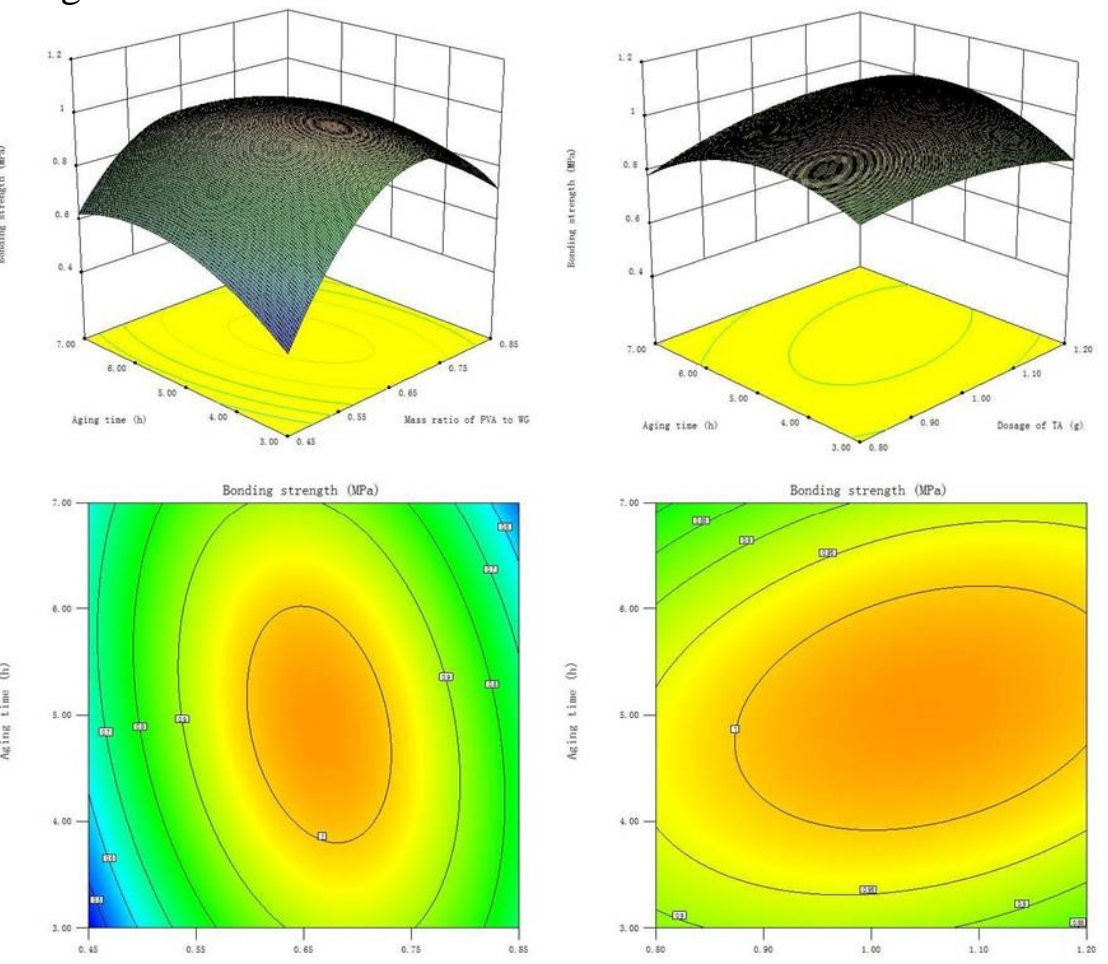

(b) $X_{1}$ and $X_{3}$

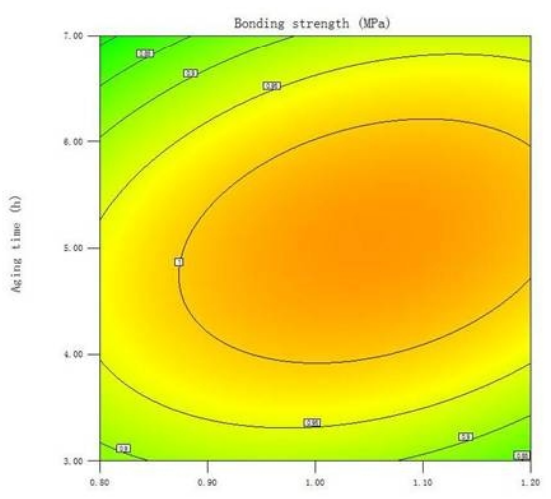

(c) $\mathrm{X}_{2}$ and $\mathrm{X}_{3}$

Fig. 1 Response surface and contour plots of the interaction of any two factors on the bonding strength value

Response surface and contour plots of the interaction of any two factors on the bonding strength are shown in Fig. 1. For Fig. 1 (a), the apparent oval contour showed very strong interactions between $X_{1}$ and $X_{2}$. The bonding strength the increase first and then decreased with the increasing $X_{1}$, the 
bonding strength reached the maximum when $X_{1}$ was about 0.65 , at the same time the bonding strength firstly increased then decreased with the increasing $\mathrm{X}_{2}$.

For Fig. 1 (b), it can be found a strong interaction between $X_{1}$ and $X_{3}$ from the oval contour. The bonding strength increased with the increasing $\mathrm{X}_{3}$, but $\mathrm{X}_{3}$ was limited, which was also affected by $\mathrm{X}_{2}$, so $\mathrm{X}_{3}$ had an optimal time of $5.00 \mathrm{~h}$ at a fixed value of $\mathrm{X}_{2}$. It can be seen from Fig. 1 (c), the bonding strength increased with the increasing $X_{3}$, it reached a maximum when $X_{3}$ was $5.00 \mathrm{~h} . \mathrm{X}_{3}$ continues to increase, the bonding strength decreased because too long $X_{3}$ would lead to the excessive crosslinking of PVA.

Analog optimization and testing. It was found that $\mathrm{X}_{1}, \mathrm{X}_{2}$, and $\mathrm{X}_{3}$ had maximum points from Fig.1, the optimal preparation conditions could be obtained accordingly using the response surface methodology. That is, the mass ratio of PVA to WG is 0.67 , the dosage of TA is $1.07 \mathrm{~g}$ and the aging time is $5.01 \mathrm{~h}$. The forecasted bonding strength is $1.0407 \mathrm{MPa}$. Considering the actual experimental conditions, the above-described conditions can be amended to as follows: the mass ratio of PVA to WG is 0.67 , dosage of TA is $1.00 \mathrm{~g}$, and aging time is $5.00 \mathrm{~h}$. Validated tests were carried out three times in accordance with the optimized conditions; the average bonding strength was $1.01 \mathrm{MPa}$, which was consistent with the predicted value, indicating the model is reasonable and effective.

Characterization of PVA-WG adhesive. As shown in Fig. 2, the FTIR of PVA-WG adhesive contained almost all of the peaks at FTIR of PVA and WG, additionally in the FTIR of PVA-WG adhesive, there are the stretching vibration peak of $\mathrm{Si}-\mathrm{OH}$ at $3452 \mathrm{~cm}^{-1}$, the characteristic peaks of $\mathrm{CH}_{2}$ - at $2926 \mathrm{~cm}^{-1}$, the stretching vibration peak of Si-O-Si at $1590 \mathrm{~cm}^{-1}$, the stretching vibration peak of $\mathrm{C}=\mathrm{O}-\mathrm{O}$ at $1072 \mathrm{~cm}^{-1}$, and the bending vibration peak of $\mathrm{Si}-\mathrm{OH}$ at $844 \mathrm{~cm}^{-1}$, indicating PVA was successfully combined into WG molecule.

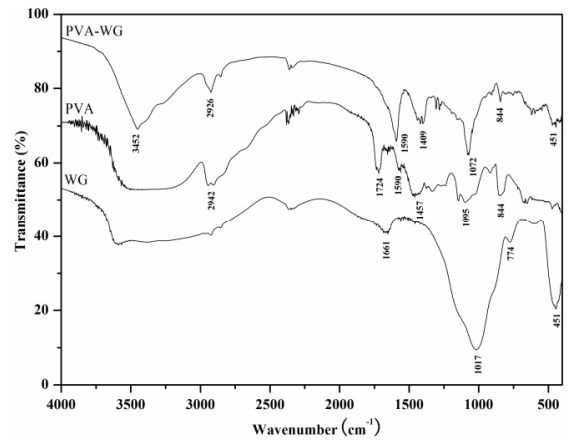

Fig. 2 FTIR of PVA, WG and PVA-WG adhesive

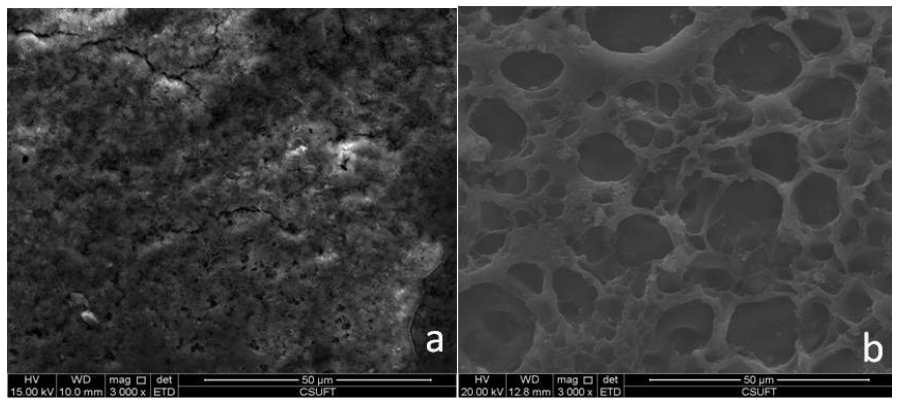

Fig. 3 SEM images of WG and PVA-WG adhesive: (a) WG; (b) PVA-WG

The effect of PVA on the adhesion structure was directly shown by SEM photographs in Fig. 3. Under the condition of the same magnification, the cured film of WG was fragile, and some brittle cracks also appeared on the surface of the film. Compared with WG, the cured film of PVA-WG adhesive was more flexible and many mesh structure formed on the surface, showing that PVA improved the toughness of WG, changed the state of aggregation of WG, and semi-interpenetrating network formed between PVA and WG under the chemical action of acids. 

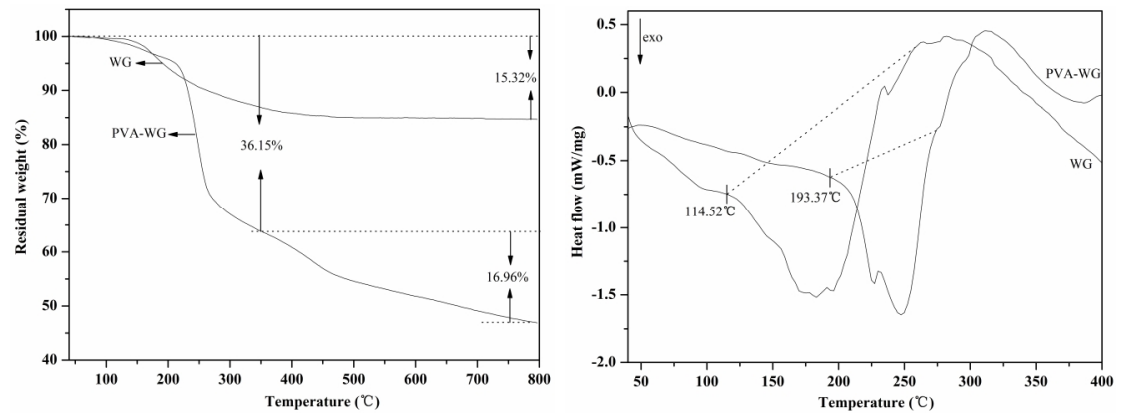

Fig. 4 TGA and DSC curves of WG and PVA-WG adhesive

The thermal properties of WG before and after modification were tested and the results were presented in Fig.4. From the TGA curves on the left of Fig.4, it could be found WG had higher thermal stability than PVA-WG, the total weight loss of WG was $15.32 \%$, but that of PVA-WG was $53.11 \%$. The modification reduced the thermal stability of WG, but the residual weight of PVA-WG was $46.89 \%$ at $800^{\circ} \mathrm{C}$, indicating PVA-WG had good thermal stability to meet the requirements for the use of wood adhesive. From the DSC curves on the right of Fig.4, the initial curing temperature of WG and PVA-WG was $114.52^{\circ} \mathrm{C}$ and $193.37^{\circ} \mathrm{C}$, respectively. The initial curing temperature of PVA-WG was $78.85^{\circ} \mathrm{C}$ higher than that of WG, showing that WG began to cure at lower temperature, which was consistent with the experimental facts.

\section{Conclusions}

(1) The experimental results and analysis showed that the preparation technology of PVA-WG adhesive can be optimized by response surface methodology, the obtained regression equation is $Y=1.03+0.044 X_{1}+0.025 X_{2}-0.00375 X_{3}+0.090 X_{1} X_{2}-0.10 X_{1} X_{3}$ $+0.045 X_{2} X_{3}-0.34 X_{1}^{2}-0.051 X_{2}^{2}-0.12 X_{3}^{2}$

ANOVA showed that the fitting of the test was significant, the determination coefficient $R^{2}=0.9701$, which can predict the change rule of bonding strength with each factor.

(2) The optimized preparation condition of PVA-WG was as follows: the mass ratio of PVA to WG was 0.65 , dosage of TA was $1.00 \mathrm{~g}$ and aging time was $5.00 \mathrm{~h}$, the bonding strength was $1.0407 \mathrm{MPa}$, close to the actual value of $1.01 \mathrm{MPa}$.

(3) The introduction of PVA changed the structure of WG adhesive, thus enhanced the performance of bonding, but the thermal stability of WG adhesive was reduced and the initial curing temperature was increased after the modification.

\section{Acknowledgements}

This work was financially supported by National Natural Science Foundation of China (31300485) and the Special Foundation for Young Core Teachers of Hunan Province (2014).

\section{References}

[1] A.Q. Cyrud, T. Bringslimark and F. Englund: Eur. J. Wood Prod., 2012, 70, (4), 541-543.

[2] Z.J. Wang, Z.B. Gu, Y. Hong, L. Cheng and Z.F. Li: Carbohydr. Polym., 2011, 86, (1), 72-76.

[3] S.W. Choi, D.W. Seo, Y.D. Lim, Y.G. Jeong, M.S. Islam Mollah, H. Park, T.W. Hong and W.G. Kim: J. Appl. Polym. Sci., 2011, 121, (2), 764-769.

[4] H.G. Silverman and F.F. Roberto: Mar. Biotechnol., 2007, 9, 6, 661-681.

[5] M. Sedano-Mendoza, P. Navarrete and A. Pizzi: Eur. J. Wood Prod., 2010, 68, (6), 355-357. 
[6] Y. Wang, K.Q. Guo, J.N. Li, X.F. Duan and Y.J. Li: Chinese Wood Ind., 2009, 23, (2), 13-15.

[7] M.A. Khan and S.M. Ashraf: Indian J. Chem. Techn., 2006, 13, (5), 347-352.

[8] H.D. Kim: J. Appl. Polym. Sci., 2007, 104, (6), 3663-3669.

[9] L.F. Zhao, Y. Liu, Z.D. Xu, Y.Z. Zhang, F. Zhao and S.B. Zhang: For. Stud. China, 2011, 13, (4), 321-326.

[10] X.L. Zhang, Y.Q. Wu, S.L, Yang and X.M. Liu: Mate. Res. Innov., 2014, 18, (S2), 532-536.

[11] X.M. Liu, Y.Q. Wu, X.L. Zhang and Y.F. Zuo: Bioresources, 2015, 10, (1): 1528-1542.

[12] Z. Lin: Master thesis of Fujian Agriculture and Forestry University (Chinese), April 2013, P58. 